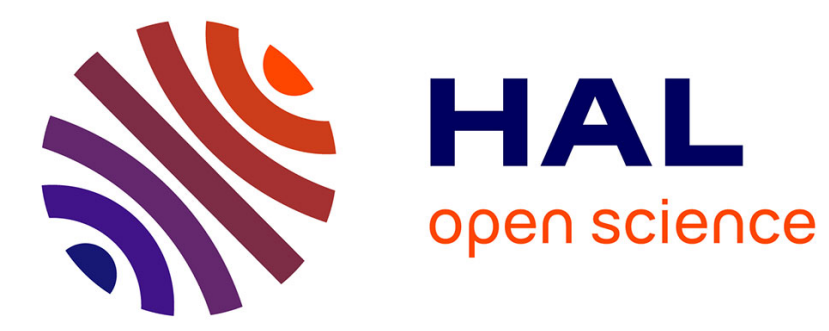

\title{
$\alpha$-Hemolysin membrane pore density measured on liposomes
}

Joel Lemiere, Karine Guevorkian, Clément Campillo, C. Sykes, Timo Betz

\section{To cite this version:}

Joel Lemiere, Karine Guevorkian, Clément Campillo, C. Sykes, Timo Betz. $\alpha$-Hemolysin membrane pore density measured on liposomes. Soft Matter, 2013, 9, pp.3181-3187. 10.1039/c3sm27812c . hal-01319268

\section{HAL Id: hal-01319268 \\ https://hal.science/hal-01319268}

Submitted on 3 Jul 2016

HAL is a multi-disciplinary open access archive for the deposit and dissemination of scientific research documents, whether they are published or not. The documents may come from teaching and research institutions in France or abroad, or from public or private research centers.
L'archive ouverte pluridisciplinaire HAL, est destinée au dépôt et à la diffusion de documents scientifiques de niveau recherche, publiés ou non, émanant des établissements d'enseignement et de recherche français ou étrangers, des laboratoires publics ou privés. 


\title{
Soft Matter
}

RSCPublishing

\section{$\alpha$-Hemolysin membrane pore density measured on liposomes}

\author{
Joël Lemière, ${ }^{\text {abcd }}$ Karine Guevorkian, ${ }^{\text {abc }}$ Clément Campillo, $^{\text {abc }}$ Cécile Sykes $^{\text {abc }}$ \\ and Timo Betz ${ }^{* a b c}$
}

Membrane pore proteins are powerful tools that allow manipulation of the inside composition of micron sized bioreactors such as artificial liposomes. While the pores self-assemble very reliably on phospholipid bilayers, the determination of the number of pores in situ for liposomes remains difficult. Here we present three independent methods to establish the number of pores on different types of liposomes:

Received 16th July 2012

Accepted 9th January 2013

DOI: $10.1039 / \mathrm{c} 3 \mathrm{sm} 27812 \mathrm{c}$

(A) the loss of refractive index due to equilibration of the inside and outside buffer conditions, and the loss of volume by (B) membrane aspiration and by (C) membrane tether pulling experiments. With these three methods we are able to determine the pore density on the membrane, and all measurements give www.rsc.org/softmatter similar values; an average pore distance is found on the order of $100 \mathrm{~nm}$.

\section{Introduction}

During the past decades, artificial phospholipid bilayer liposomes have been extensively studied. One major research theme was the possibility of using these liposomes as microscopic reactors for biomimetic systems. ${ }^{1,2}$ The advantage of artificial liposomes is the possibility of controlling both the lipid bilayer composition and the inside content. This allows reconstitution of certain aspects of living biological systems, and even rebuilding of certain properties of living cells. Recent advances have shown that it is possible to control the liposome inside solution up to a degree where protein construction via ribosomes is possible, ${ }^{2}$ or to even reconstitute the actin cortex of living cells by inducing actin polymerization at the inner membrane. ${ }^{1}$ Two important liposome preparation techniques are electroformation and the recently developed inverted emulsion technique. While the electroformation is, today, a simple and common technique, the inverted emulsion method is more complex but allows encapsulation of a well defined and precious content within the liposomes while having a totally different solution outside. In this report we will investigate both formation methods.

Many applications of liposome micro-reactors require a controlled triggering of the inside reactions and a steady control of parameters such as salt concentration, $\mathrm{pH}$ or the supply of energy in the form of adenosine triphosphate (ATP). This problem was solved by the addition of membrane pores with a well defined pore diameter, thus allowing the exchange of

\footnotetext{
aInstitut Curie, Centre de Recherche, F-75248, Paris, France.E-mail: timo.betz@curie. fr

${ }^{b}$ CNRS, UMR168, F-75248, Paris, France

'UPMC Univ Paris 06, UMR 168, F-75005, Paris, France

${ }^{d}$ Univ. Paris Diderot, Sorbonne Paris Cité, F-75205, Paris, France
}

molecules below a mass threshold, while preserving larger molecules such as proteins inside the liposome. A common pore protein used for that is the bacterial toxin $\alpha$-hemolysin, which is secreted by the bacteria Staphylococcus aureus as a water soluble monomer with a size of $33.2 \mathrm{kDa}^{3-5}$ Monomers self-assemble spontaneously on a phospholipid bilayer to form a mushroom-shaped pore with a cutoff mass of about $2 \mathrm{kDa}$, which is permeable to ions and single nucleotides, but blocks larger proteins that do not fit through the $1.5 \mathrm{~nm}$ pore diameter. ${ }^{3}$ The $\alpha$-hemolysin pore therefore satisfies the mentioned requirement to control $\mathrm{pH}$, ions and ATP-concentration, while confining proteins inside the liposomes. Furthermore, the pore properties can be well controlled by genetic modifications. ${ }^{6}$ However, the quantification of the pore insertion density has remained a complex problem, which was commonly ignored as the pore protein was added in high concentrations to simply result in a saturation of pores on the membrane. Nevertheless, a good estimation of the pore concentration is important to understand the dynamics of the molecular exchange and to estimate the material fluxes of molecules consumed inside the liposome, such as ATP or amino acids. ${ }^{2}$ Commonly, the presence of pores is measured by the ion current flow, when applying a membrane potential. However, this method only works well for a small number of pores that are generally situated on a small membrane patch that is aspirated into a micropipet. ${ }^{6}$ On liposomes, this technique is not possible as it requires the insertion of an electrode into the liposome, which ruptures the membrane and thus destroys the liposome.

Here we address the question of pore density by presenting three different methods, which give consistent results. Interestingly, we find that the equilibration of different insideoutside conditions might take several minutes during which the system is out-of-equilibrium. 


\section{Methods}

\subsection{Liposome preparation}

Liposome composition. EPC, DOGS-NTA-Ni, and DSPEPEG(2000)-biotin (all purchased from Avanti Polar Lipids) are mixed with a molar ratio of EPC/DOGS-NTA-Ni/biotin of $91: 8: 1$ (EPC/NTA).

Buffer composition. To image liposomes in phase contrast microscopy, different inside and outside buffers are used.

The inside buffer (I-Buffer-1) consists of $6.5 \mu \mathrm{M}$ G-actin (including $20 \%$ fluorescently labeled actin), $10 \mathrm{mM}$ HEPES (pH 7.6), $0.1 \mathrm{mM} \mathrm{CaCl}_{2}, 100 \mathrm{mg} \mathrm{ml}{ }^{-1}$ dextran, $0.2 \mathrm{mM} \mathrm{ATP,} 6 \mathrm{mM}$ dithiothreitol (DTT), $0.13 \mathrm{mM}$ diazabicyclo[2,2,2]octane (Dabco), and $280 \mathrm{mM}$ sucrose. The outside buffer (O-Buffer-1) contains $10 \mathrm{mM}$ HEPES (pH 7.5), $2 \mathrm{mM} \mathrm{MgCl} 2,0.2 \mathrm{mM} \mathrm{CaCl}_{2}$, $2 \mathrm{mM}$ ATP, $6 \mathrm{mM}$ DTT, $0.13 \mathrm{mM}$ Dabco, $275 \mathrm{mM}$ glucose, and $0.5 \mathrm{mg} \mathrm{ml} \mathrm{ml}^{-1}$ casein. These buffers were used in liposome aspiration and tube pulling experiments. The inside buffer (I-Buffer-2) consists of $300 \mathrm{mM}$ sucrose. The outside buffer (O-Buffer-2) contains $300 \mathrm{mM}$ glucose and $0.5 \mathrm{mg} \mathrm{ml}^{-1}$ casein. Contrast loss measurements are applied on liposomes prepared with these buffers.

Inverted emulsion. ${ }^{1}$ Lipids are dissolved in mineral oil (Sigma) at a total concentration of $0.5 \mathrm{mg} \mathrm{ml}^{-1}$. A volume of $5 \mathrm{ml}$ of this oil-lipid mixture is sonicated in a bath at 35 degrees for $30 \mathrm{~min}$ at a power of $30 \mathrm{~W}$, cooled to room temperature and then stored at 4 degrees for up to a week. An emulsion between the inside buffer and the oil-lipid mixture is prepared and added on top of the outside buffer. Subsequent centrifugation transports the inside solution emulsion across a lipid monolayer as described earlier ${ }^{1}$ and liposomes are collected.

Electroformation. ${ }^{7}$ Briefly, the lipid mix is dissolved in chloroform $\left(2.5 \mathrm{mg} \mathrm{ml}^{-1}\right)$ and $10 \mu \mathrm{l}$ of this solution is applied on an ITO glass slide. The slides are stored in vacuum for $1 \mathrm{~h}$ to evaporate the solvent. Subsequently, two slides are assembled into a chamber of width $1 \mathrm{~cm}$ and height $2 \mathrm{~mm}$. The cavity is filled with the inside buffer. An alternating electric field $(10 \mathrm{~Hz}$, $2.3 \mathrm{~V}$ ) is applied across the chamber for 8 hours. Liposomes created this way are diluted in the external buffer and stored at 4 degrees for up to a week.

$\boldsymbol{\alpha}$-Hemolysin. The pore-forming protein $\alpha$-hemolysin (Sigma) is dissolved to a final concentration of $1 \mathrm{mg} \mathrm{ml}^{-1}$ in a buffer containing $150 \mathrm{mM} \mathrm{KCl}$ and $10 \mathrm{mM}$ HEPES. To achieve comparable results, we always used the same final concentration of $0.1 \mathrm{mg} \mathrm{ml}^{-1} \alpha$-hemolysin in the three different experiments described here.

\subsection{Contrast measurement for the refractive index difference}

To determine the relative difference of the refractive index between inside and outside a liposome, we use a recently developed method $^{8,9}$ based on light refraction across the membrane. A laser focus positioned at the interface of a liposome filled with a higher refractive index buffer refracts the light as a function of the refractive index differences. We record this asymmetry in the far field of the laser using a quadrant photodiode (QPD) (see Fig. 1A) mounted on an Olympus IX-71 microscope stand equipped with a $\times 60$ water immersion objective (NA 1.2). During the experiments the liposome is fixed with three beads in a multiple optical tweezer system. Each bead is trapped by an effective power of $\simeq 5$ to $7 \mathrm{~mW}$. Meanwhile, we scan the focus of a weak (power $\leq 0.5 \mathrm{~mW}$ ) IR laser of wavelength $1064 \mathrm{~nm}$ over the membrane and measure the diffracted light using a QPD in the back-focal plane of the condenser. This scan results in a curve as shown in Fig. 2A that is similar to the one predicted and measured on scans over
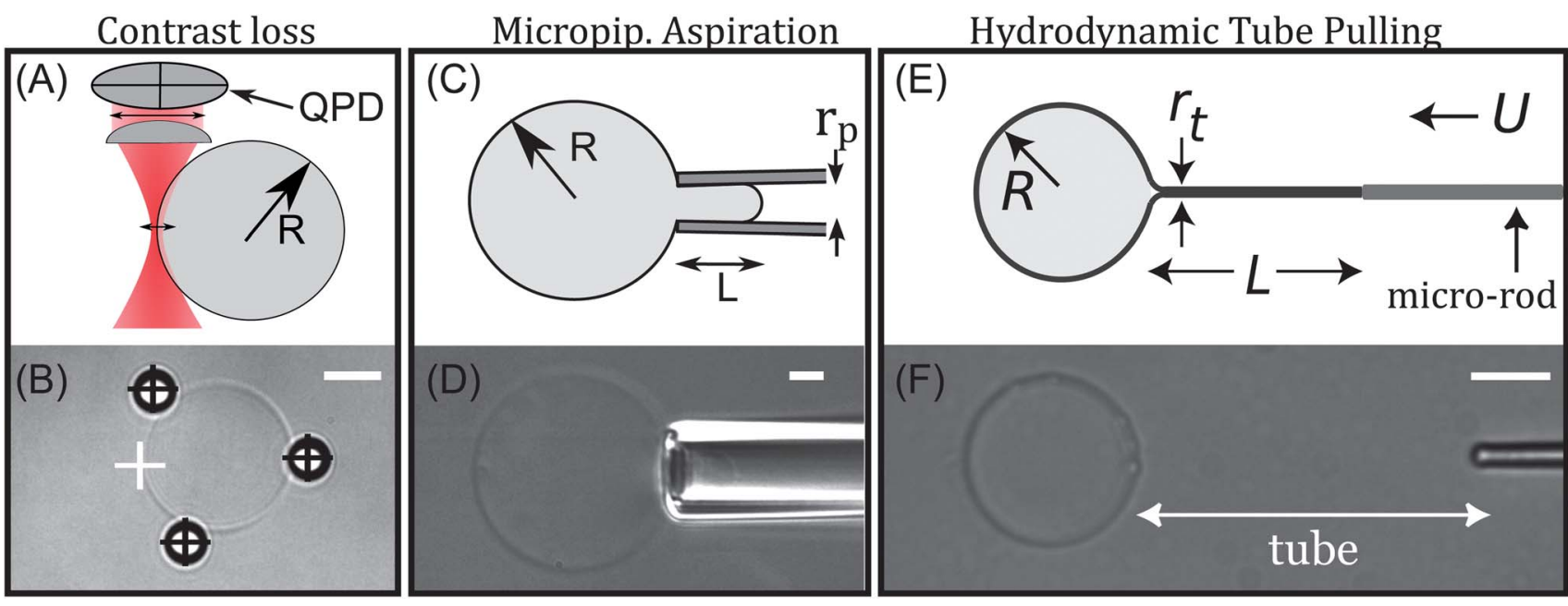

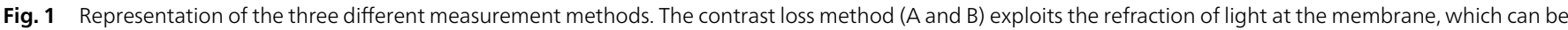

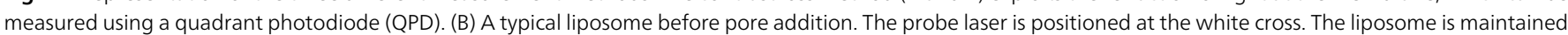

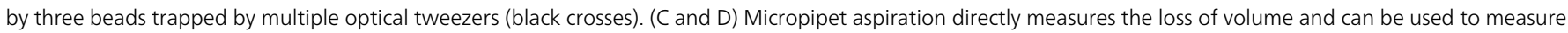

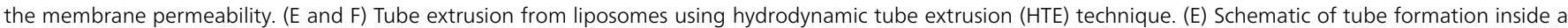

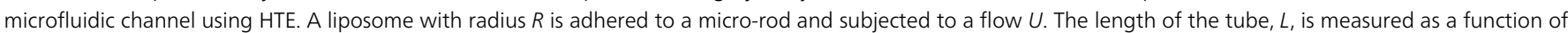
time. $r_{\mathrm{t}}$ is the radius of the tube. (F) Snapshot of a tube formed from a liposome using HTE. Scale bars are $5 \mu \mathrm{m}$. 


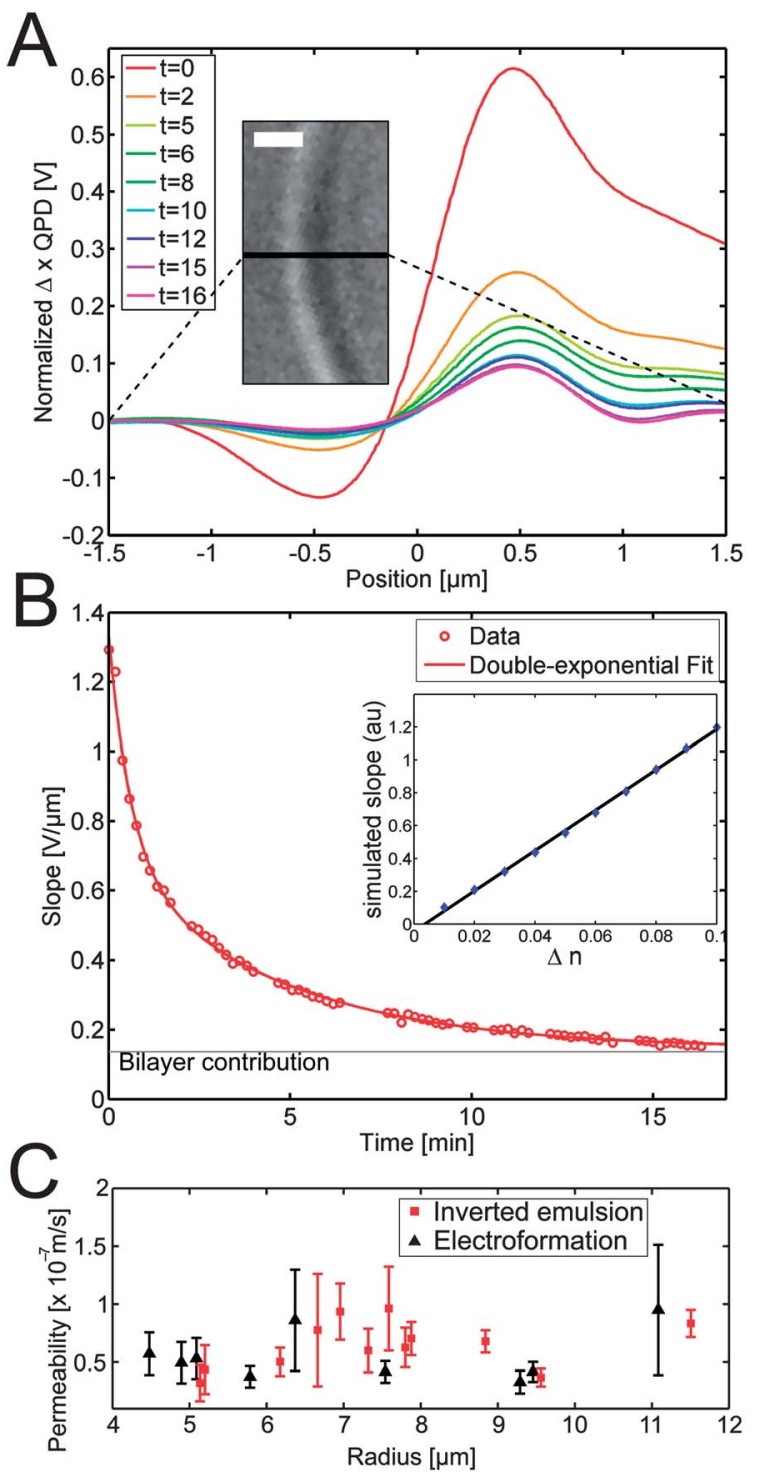

Fig. 2 (A) Decrease of signal recorded at the QPD after addition of membrane channels $\alpha$-hemolysin, time $(t)$ is given in minutes. The signal represents the scan in the $x$ direction (black line in the inset) of the diffracted light normalized by the sum signal of the QPD. The position is relative to the membrane. The inset represents an image of the membrane with the position of the laser during the scan (black line), scale bar is $1 \mu \mathrm{m}$. (B) The decay of the slope quantifies the loss of refractive index differences, and a double exponential decay function (red line) monitors the equilibration of the different molecules. Inset: numerical simulations establish the linear relation between the slope of the scan curve and the refractive index difference. Data points are simulations, and the line is a linear fit. (C) Sucrose permeability as a function of liposome radius prepared by electroformation (black triangle) or inverted emulsion (red square).

polystyrene beads. ${ }^{10}$ According to the Lorenz-Mie theory the slope of the scan curve depends linearly on the refractive index difference. The numerical solution of the Lorenz-Mie theory ${ }^{11}$ confirms the linear dependence of the slope $s$ on the refractive index difference $\Delta n$ as $s(t)=\gamma \times \Delta n$, where $\gamma$ is a complex function of the geometry and the optical configuration (inset Fig. 1B). To analyse the data, the linear dependence of the slope on the refractive index difference is used. We measure the change in refractive index difference by recording the slope of the curve along the edge of the liposomes, while the concentration of glucose and sucrose equilibrates.

\subsection{Liposome aspiration}

Liposomes are aspirated as shown in Fig. 1C and D. Pipets are pulled from glass capillaries using a micropipet puller (P2000, Sutter Instruments, USA). The opening of the pipet is cleaved to a diameter of 3-5 $\mu \mathrm{m}$ by a microforge (MF-830, Narishige, Japan). Micropipets are filled with glucose buffer and mounted on the experimental chamber. Micropipets and experimental chambers are passivated by applying a $5 \mathrm{mg} \mathrm{ml} \mathrm{m}^{-1}$ casein solution for 30 minutes. Micropipette aspiration experiments are performed on a Nikon TE2000 inverted microscope with a $\times 100 /$ NA 1.3 oil immersion objective (Nikon). To vary the pressure inside the micropipet, it is connected to a water reservoir of variable height using a tube system. Before each experiment, the point of zero pressure between the micropipet and the chamber is measured by adjusting it to the point of zero flow by aspirating tracer particles that are observed in the micropipet.

\subsection{Hydrodynamic tube pulling}

The experimental chamber consists of a single microfluidic channel made out of poly(dimethylsiloxane) (PDMS), which is attached to a cover-glass through plasma activation. One side of the channel is cut open to allow the introduction of the liposomes and the glass micro-rod. The other end is connected to a syringe pump (KDS Scientific, USA), which operates in aspiration mode. The details of the setup are explained elsewhere. ${ }^{12}$ The channel is $200 \mu \mathrm{m}$ high, $600 \mu \mathrm{m}$ wide and $2 \mathrm{~cm}$ long. A coated glass micro-rod is used to attach the liposomes and to pull a tether under medium flow. Thin-tip micro-rods are prepared by pulling borosilicate rods (1 $\mathrm{mm}$ outer diameter) using a laser-based puller (P-2000, Sutter Inst., USA). The rods are then forged to $2-3 \mu \mathrm{m}$ using a micro-forge (Narishige, Japan). Rods are coated with streptavidin to attach the membrane to the glass. This is done by first cleaning them in plasma cleaner for 30 seconds, then incubating them in $0.1 \%$ $\mathrm{v} / \mathrm{w}$ poly-lysine (Sigma) for 30 minutes. Then they are rinsed with PBS and incubated in $2.5 \mathrm{mg} \mathrm{ml}^{-1}$ streptavidin (Sigma) in PBS solution for 1 hour. The adhesion of the liposomes to the glass micro-rod takes place through the streptavidin on the micro-rod and the biotinylated lipids in the liposome membrane. Once the liposome is attached to the rod, it is brought into the channel using a mechanical micro-manipulator and raised to the middle of the channel, see Fig. 1E and F. Using the syringe pump in aspiration mode a fixed debit, $D_{\mathrm{f}}$, is applied. The flow velocity has a Poiseuille distribution, is strongest at the mid-height of the channel, and is related to $D_{\mathrm{f}}$ as: $U=1.5 \times D_{\mathrm{f}} / A_{\mathrm{c}}$, where $A_{\mathrm{c}}$ is the cross-section of the channel. The tubes are extruded after equilibration of the sucroseglucose gradient through the $\alpha$-hemolysin pores. Liposomes are visualized in epifluorescence using an inverted microscope (Zeiss Axiovert 100) equipped with a $\times 20$ air objective (NA 0.45 ). Time lapse movies are recorded with a CDD camera (Luca-R, Andor) at $200 \mathrm{~ms}$ intervals. Traction of the liposomes is performed using ImageJ software (NIH). 


\section{Results and discussion}

\subsection{Time dependent refractive index difference}

Before the incorporation of pores into the lipid bilayer, liposomes are visible in bright-field microscopy due to the difference in the refractive indices of sucrose and glucose. The addition of pores allows equilibration of the glucose-sucrose concentrations across the membrane. This in turn reduces the asymmetry measured by the QPD. To quantify the loss of contrast we use the linear region of the scan curve as shown in Fig. $2 \mathrm{~A} .{ }^{\mathbf{1 1}}$ The slope of the linear regime between the minimal and the maximal signal is found by fitting a line along the 200 $\mathrm{nm}$ region centered around the midpoint between the curve minimum and maximum.

Before pore addition, the deflection is determined by the refractive indices of the inside buffer and outside buffer, $n_{\mathrm{I}}=$ 1.348 and $n_{\mathrm{O}}=1.344$, respectively. The initial refractive index difference of $\Delta n=0.004$ decreases while the concentration equilibrates across the membrane through the $\alpha$-hemolysin pores. To measure the permeability, which allows estimation of the pore density, we will only require the time dependence of $\Delta n$ that is proportional to the slope $s$ (see Material and methods).

Fig. 2B shows the decrease of the slopes over time, starting right after the addition of pores. The equilibration of the glucose-sucrose decreases the slope $s$ until the point where only the refraction of the lipid bilayer is left. The contribution of the pure membrane is detectable $\left(0.15 \mathrm{~V} \mathrm{~mm}^{-1}\right)$ and constant as shown in Fig. 2A and B.

In the following, we will use the fact that for small concentrations, the refractive index is a linear function of the concentration of molecules in solution; hence $n$ is proportional to $c$ and we need to consider only the concentration change over time to model the data. Here we can make use of the known behaviour of concentration equilibration due to the permeability $\alpha$, which contributes to the permeability equation:

$$
\frac{\mathrm{d} c^{\mathrm{i}}}{\mathrm{d} t}=-\frac{\alpha A}{V} \Delta c
$$

where $c^{\mathrm{i}}$ is the concentration inside the liposome, $\alpha$ is its permeability, $A$ is the liposome surface, and $\Delta c=c^{\mathrm{i}}-c^{\mathrm{ext}}$ the concentration difference across the membrane. Furthermore, since we know that the outside concentration stays constant due to the large volume, $\mathrm{d} c^{\mathrm{i}} / \mathrm{d} t=\mathrm{d}(\Delta c) / \mathrm{d} t$ and thus, knowing the radius $R$ of the liposome:

$$
\frac{\mathrm{d}(\Delta c)}{\mathrm{d} t}=-\frac{3 \alpha}{R} \Delta c
$$

gives the solution:

$$
\Delta c(t)=\Delta c_{0} \exp \left(-\frac{3 \alpha}{R} \times t\right)
$$

where $\Delta c_{0}$ is the initial concentration difference. In fact, this calculation needs to be done for both molecular species, sucrose and glucose, marked respectively with the indices s and g:

$$
\Delta c^{\mathrm{s}, \mathrm{g}}(t)=\Delta c_{0}^{\mathrm{s}, \mathrm{g}} \exp \left(-\frac{3 \alpha_{\mathrm{s}, \mathrm{g}}}{R} \times t\right)
$$

The refractive index difference can be written as:

$$
\Delta n=\left(n_{\mathrm{i}}-n_{\mathrm{ext}}\right)+\Delta n_{\mathrm{M}}
$$

with $n_{\mathrm{i}}$ and $n_{\text {ext }}$ as the inside and outside refractive indices respectively and $\Delta n_{\mathrm{M}}$ the membrane contribution, we can rewrite the expression using $\beta$ as an unknown proportionality constant between concentration and refractive index to yield:

$$
\begin{gathered}
\Delta n(t)=\left(n_{\mathrm{i}}^{\mathrm{s}}(t)+n_{\mathrm{i}}^{\mathrm{g}}(t)\right)-\left(n_{\mathrm{ext}}^{\mathrm{s}}+n_{\mathrm{ext}}^{\mathrm{g}}\right)+\Delta n_{\mathrm{M}} \\
\Delta n(t)=\Delta n^{\mathrm{s}}(t)+\Delta n^{\mathrm{g}}(t)+\Delta n_{\mathrm{M}} \\
\Delta n(t)=\beta \Delta c^{\mathrm{s}}(t)+\beta \Delta c^{\mathrm{g}}(t)+\Delta n_{\mathrm{M}}
\end{gathered}
$$

Here we have established the connection between the total refractive index difference and the different contributions from glucose, sucrose and the membrane. As previously mentioned, the measured slope of the scan is directly proportional to the refractive index difference. Thus:

$$
s(t)=s_{\mathrm{s}} \exp \left(-\frac{3 \alpha_{\mathrm{s}}}{R} \times t\right)+s_{\mathrm{g}} \exp \left(-\frac{3 \alpha_{\mathrm{g}}}{R} \times t\right)+s_{\mathrm{M}}
$$

where $s_{\mathrm{s}}$ and $s_{\mathrm{g}}$ are simple prefactors $s_{\mathrm{s}, \mathrm{g}}=\gamma \beta \Delta c_{0}^{\mathrm{s}, \mathrm{g}}$ that ensure the transition from concentration difference to slope. For the following we are exclusively interested in the time dependence of eqn (9), which yields the permeabilities $\alpha_{\mathrm{s}, \mathrm{g}}$. The last term accounts for the contribution of the lipid bilayer that is unaffected by the change of molecules. Fig. 2B shows the slope contribution of the pure membrane $s_{\mathrm{M}}=0.1453 \pm 0.01 \mathrm{~V} \mathrm{~nm}^{-1}$.

As seen in Fig. 2B, experimentally we find a slow and a fast exponential decay. We can attribute the fast decay to the equilibration of the smaller molecule, glucose, $\alpha_{\mathrm{g}}=1.1 \pm 0.1 \times$ $10^{-7} \mathrm{~m} \mathrm{~s}^{-1}$ and slow decay to sucrose, $\alpha_{\mathrm{s}}=1.5 \pm 0.2 \times 10^{-8} \mathrm{~m}$ $\mathrm{s}^{-1}$. Commonly, the fast decay is less accurate; hence we exclusively use the slow equilibration of sucrose for the analysis presented in this work, which is represented by the first term of eqn (9). Note that this difference in the speeds of glucose and sucrose molecules leads to an intermediate timescale where the effective tension will increase as more glucose has entered the liposome than sucrose has left.

We perform multiple experiments with this technique using both vesicles made by electroformation (EF) or inverted emulsion (IE). Fig. 2C shows the dependence of the permeability coefficient $\alpha_{\mathrm{s}}$ with the radius of liposomes for the two preparation methods. No significant dependence of $\alpha_{\mathrm{s}}$, neither on the radius nor on the preparation method is observed. These measurements yield a permeability of sucrose for inverted emulsion $\alpha_{\mathrm{s}}(\mathrm{IE})=6.5 \pm 2 \times 10^{-8} \mathrm{~m} \mathrm{~s}^{-1}$ and electroformation $\alpha_{\mathrm{s}}(E F)=5.5 \pm 2 \times 10^{-8} \mathrm{~m} \mathrm{~s}^{-1}$, which shows that both methods are consistent as they have no significant difference $\left(p_{\text {value }}=\right.$ $0.27 \gg 0.05)$.

Taking into account the high concentration of membrane proteins, these data fit well with previous measurements of $\alpha$-hemolysin-mediated permeability on fibroblasts where salt permeabilities of $10^{-9}$ to $10^{-8} \mathrm{~m} \mathrm{~s}^{-1}$ have been measured for a small concentration of pores $\left(1-50 \mu \mathrm{g} \mathrm{ml}^{-1}\right.$, compared to $100 \mu \mathrm{g}$ $\mathrm{ml}^{-1}$ in the current experiments). ${ }^{13}$ 
Table 1 Resulting pore density, $\rho$, average pore distance $\bar{d}$ and number of experiments $N$ for the three different experimental approaches: contrast loss, micropipet aspiration and hydrodynamic tube pulling. Note that for EPC the error represents the $95 \%$ confidence interval of the fit

\begin{tabular}{llcrr}
\hline Method & Lipids & $\rho\left(\mu \mathrm{m}^{-2}\right)$ & $\bar{d}(\mathrm{~nm})$ & $N$ \\
\hline Contr. loss (EF) & EPC/NTA & $125 \pm 45$ & $90 \pm 16$ & 9 \\
& EPC & $34 \pm 5$ & $171 \pm 12$ & 1 \\
Contr. loss (IE) & EPC/NTA & $147 \pm 45$ & $82 \pm 12$ & 12 \\
& EPC & $24 \pm 5$ & $208 \pm 22$ & 1 \\
Microp. aspiration & EPC/NTA & $122 \pm 69$ & $90 \pm 30$ & 4 \\
Hyd. tube pulling & EPC/NTA & $146 \pm 67$ & $83 \pm 30$ & 4 \\
& & & & \\
\hline
\end{tabular}

To calculate the density of pores on the surface of the liposomes, we use the established model of diffusion through small pores by Pappenheimer ${ }^{14}$ and Renkin. ${ }^{15}$ For a pore of area $A_{0}=$ $\pi r^{2}$ (with $r$ as pore radius), the diffusion depends on the size of the passing molecule, which is defined by the hydrodynamic radius $a$. Pappenheimer and Renkin showed that the hydrodynamic drag can be taken into account when the actual pore area $A_{0}$ is replaced by an effective pore area $A_{\mathrm{p}}$, defined as: $A_{\mathrm{p}} / A_{0}=$ $(1-(a / r))^{2} \times\left[\left(1-2.104(a / r)+2.09(a / r)^{3}-0.95(a / r)^{5}\right]\right.$. The permeability coefficient depends on the pore density $\rho$, the diffusion constant $D=k_{\mathrm{B}} T /(6 \pi \eta a)$ and the length of the pore $l$, and is described by $\alpha=\rho D A_{\mathrm{p}} / l$. Using these expressions we can calculate the pore density from the measured permeability by:

$$
\rho=\frac{\alpha l}{A_{\mathrm{p}}} \times \frac{6 \pi \eta a}{k_{\mathrm{B}} T}
$$

with $A_{\mathrm{p}}$ as defined above.

The molecular parameters of the $\alpha$-hemolysine channel have been measured previously to yield a pore radius of $r=0.7 \mathrm{~nm}$ and a pore length of $l=10 \mathrm{~nm} \cdot{ }^{3,4}$ Furthermore, the hydrodynamic diameter of sucrose has been determined previously, $a_{\mathrm{s}}=0.5 \mathrm{~nm} .{ }^{16} \mathrm{We}$ apply this calculation using a viscosity of $\eta=$ $1 \times 10^{-3} \mathrm{~Pa} \times \mathrm{s}$ to estimate the pore density $\rho_{\mathrm{s}}(\mathrm{EF})=124 \pm 45$ $\mu \mathrm{m}^{-2}$ for electroformed liposomes, $\rho_{\mathrm{s}}$ (IE) $=147 \pm 45 \mu \mathrm{m}^{-2}$ for the inverted emulsion and a respective mean pore distance of $\bar{d}_{\mathrm{s}}(\mathrm{EF})=90 \pm 16 \mathrm{~nm}, \bar{d}_{\mathrm{s}}(\mathrm{IE})=82 \pm 12 \mathrm{~nm}$ (Table 1$)$. It should be noted that this method directly yields average pore densities of full liposomes, as possible local concentration gradients inside the liposome equilibrate on a timescale of $\approx 150 \mathrm{~ms}$, while the timescale of sucrose/glucose equilibration across the membrane is $\mathbf{6}-\mathbf{1 2}$ minutes.

Two other experiments on a pure EPC membrane lead to about five times smaller values of the pore densities (see Table 1), which suggests a dependence of lipid compositions on pore insertion.

\subsection{Micropipet aspiration leads to a steady loss of volume}

To directly measure the loss of volume in a quasi-equilibrated situation, we aspirate liposomes with a given pressure, and use the volume loss to estimate the pore density. These experiments are performed after equilibration of the sucrose-glucose concentration between the liposome and the buffer. A constant pressure is applied, which forces a constant water flow. Assuming that water permeability increases due to the pores, we thus can estimate the pore number. This measurement does in principle depend on the correct estimation of the volume loss over time.

In each experiment, we set the aspiration pressure in the micropipet $\Delta P$ to 0 and bring the micropipet tip close to a liposome membrane. Then we apply a constant aspiration pressure $\Delta P=160 \mathrm{~Pa}$ and monitor the entry of a liposome tongue inside the micropipet. We calculate the volume $V(t)$ of the liposome from the measurement of micropipet radius $r_{\mathrm{p}}(t)$, the radius of external portion of the liposome $R(t)$ and length of the aspirated portion of the liposome $L(t)^{17}$ (see Fig. 1C):

$$
V(t)=\frac{2 \pi r_{\mathrm{p}}{ }^{3}}{3}+\pi r_{\mathrm{p}}\left(L(t)-r_{\mathrm{p}}\right)+\frac{\pi R(t)^{3}}{3}\left(2+3 u(t)-u(t)^{3}\right)
$$

where

$$
u(t)=\sqrt{1-\left(\frac{r_{\mathrm{p}}}{R(t)}\right)^{2}}
$$

Fig. 3 shows the evolution of liposome volumes with time for four examples. We observe that $V(t)$ decreases linearly with time, showing that the flow $J$ is constant for each liposome. Assuming for simplicity that most of the water is leaking though the pores, we can use the expression for the flux through $N$ pores of radius $r$ by: ${ }^{18}$

$$
J=N r^{3} \Delta P / 3 \eta_{\mathrm{i}}
$$

where $\Delta P=2 \sigma / R$, and $\eta_{\mathrm{i}}$ is the viscosity of the fluid inside. This reasoning allows the estimation of the number of pores to be $N^{\text {asp }}=6.1 \pm 3.5 \times 10^{4}$, which gives a density of $122 \pm 69$ pores per $\mu \mathrm{m}^{2}$ and an average pore distance of $90 \pm 30 \mathrm{~nm}$.

While these values are smaller that the measurements of the loss of contrast, they range in the same order of magnitude.

\subsection{Hydrodynamic membrane tube pulling}

Extrusion of membrane nanotubes from liposomes is used to probe the membrane tension, bending modulus, and

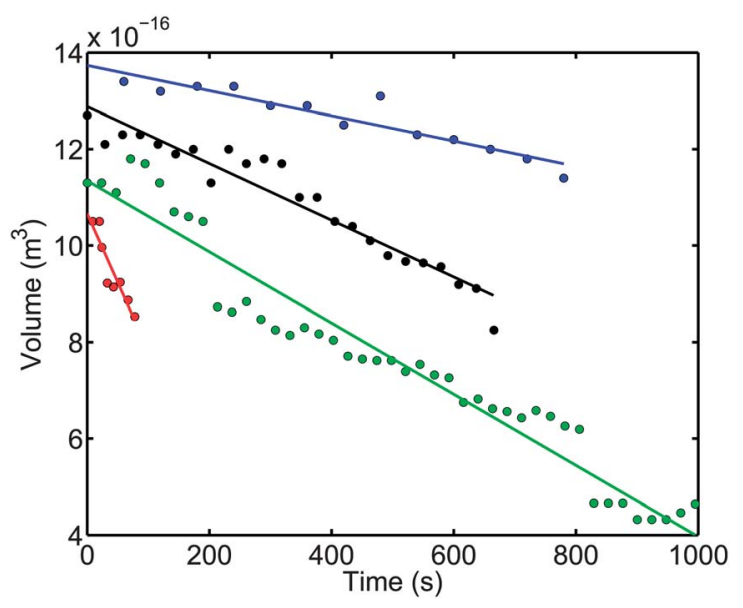

Fig. 3 Loss of volume over time for several examples measured by liposome aspiration. The lines are the linear fit functions, where all have a good fit quality ( $R$-square values between 0.85 and 0.95 ). 
permeability of these liposomes. The critical force needed to extrude a tube depends on the bending modulus of the membrane, $\kappa$ and the surface tension $\sigma$ as: $f_{\text {tube }}=2 \pi \sqrt{2 \kappa \sigma}=2 \pi \kappa / r_{\mathrm{t}}$, where $r_{\mathrm{t}}$ is the tube radius. In the hydrodynamic tube extrusion (HTE) technique, a controlled fluid flow is used to apply a force on an anchored liposome. Due to the rapid nature of this experiment, we cannot assume equilibrium of the glucose diffusion through the membrane. Therefore, we directly use the flow of water to analyze this experiment. The hydrodynamic force, which equals the drag force on the liposome, is given by: $f_{\mathrm{drag}}=6 \pi \eta R U$, where $\eta$ is the viscosity of the fluid, $R$ is the radius of the liposome and $U$ is the velocity of the flow. When $f_{\text {drag }}>f_{\text {tube }}$, a tube forms between the anchorage point and the liposome body downstream. Once the tube is formed, its dynamics is governed by the balance between the total friction force, $f_{\text {friction }}$ and $f_{\text {tube: }}$

$$
\begin{gathered}
f_{\text {friction }}=f_{\text {tube }} \\
6 \pi \eta R(U-\dot{L})=2 \pi \sqrt{2 \kappa \sigma}
\end{gathered}
$$

where $\dot{L}=\mathrm{d} L / \mathrm{d} t$ is the tube elongation velocity.

An impermeable liposome behaves like an entropic spring. As the tube elongates, the surface tension increases, leading to an augmentation of $f_{\text {tube }}$ and decrease of $\dot{L}$. When $\dot{L}=0$, the tube reaches a stationary length, $L_{\infty}$, and the tube no longer elongates. The surface tension and the bending modulus of an impermeable liposome can hence be obtained by finding $L_{\infty}$, as a function of $U$, as reported previously. ${ }^{12}$ In contrast, for permeable liposomes, the membrane tension stays constant and the tube is extruded at constant velocity without reaching a stationary length ${ }^{\mathbf{1 9}}$ (Fig. 4). The elongation of the tube is accompanied by a decrease of the volume $V$ of the liposome as the fluid inside the liposome leaks out with a rate given by: $J=-\mathrm{d} V / \mathrm{d} t=-4 \pi R^{2} d R / d t$. Using the area conservation criteria, $-8 \pi R \dot{R}=2 \pi r_{\mathrm{t}} \dot{L}$, and the expression of the flux (eqn (13)) we get the following relationship between the number of pores as a function flow velocity $U$ and tube extrusion speed $\dot{L}$ :

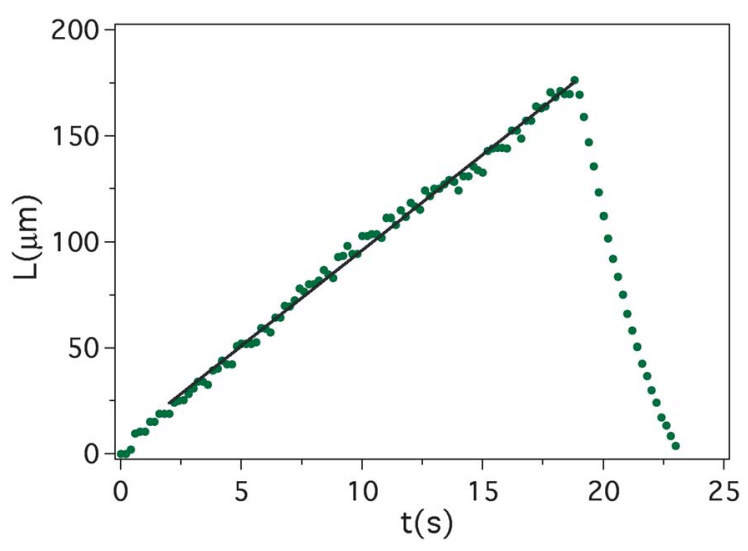

Fig. 4 Hydrodynamic tube extrusion of a liposome decorated with $\boldsymbol{\alpha}$-hemolysin pores. The tube extends with $\dot{L}=9 \mu \mathrm{m} \mathrm{s}^{-1}$, at $U=125 \mu \mathrm{m} \mathrm{s}^{-1}$ and retracts once the flow stops. The liposome radius is $R=10 \mu \mathrm{m}$.

$$
N=\frac{\pi \kappa^{2} \eta_{\mathrm{i}}}{9 \eta^{3} R r^{3}} \frac{\dot{L}}{(U-\dot{L})^{3}}
$$

Taking $r=0.7 \mathrm{~nm}$ for an $\alpha$-hemolysin pore, $\kappa=10 k_{\mathrm{B}} T$, and $\eta_{\mathrm{i}}=\eta_{\mathrm{o}}=0.9 \times 10^{-3} \mathrm{~Pa} \times \mathrm{s}$ after buffer equilibration, we obtain $N$ using eqn (15). The pore density is defined as $\rho=N / A$, where $A$ is the area of the liposome at rest. Applying this third nonequilibrium technique, we find a value of $146 \pm 67 \mu \mathrm{m}^{-2}$ for the pore density and an average distance between the pores of $83 \pm 30 \mathrm{~nm}$.

These findings match the results obtained from the micropipet aspiration measurements, and are in the same order of magnitude as the measurements obtained from the loss of contrast method.

\section{Conclusion}

We provide three different experimental ways of estimating the density of $\alpha$-hemolysin pores that auto-assemble into a liposome at a given concentration of protein. Our measurements give consistent estimates of the pore density and average pore distance, thus validating the three measurements. The different ways of experiments used the loss of refractive index after pore addition, the loss of volume during aspiration in a micropipet and the hydrodynamic extrusion of a membrane tube. Hence, we probed the initial non-equilibrium situation, the long term quasi-equilibrium during aspiration, which took up to 15 minutes, and the extension of membrane tubes, which happened on the timescale of up to 20 seconds.

The measurements of pore density and average pore distance presented show that even under variable preparation conditions, three different measurements produce comparable and consistent values that are on the order of 100 pores per $\mu \mathrm{m}^{2}$ for EPC/NTA lipids. Nevertheless, it turns out that liposomes formed by pure EPC lipids seem to have a smaller pore density as compared to vesicles formed using EPC/NTA mix lipids (Table 1). This shows that $\alpha$-hemolysin adsorption appears to be sensitive to membrane composition.

\section{Acknowledgements}

The authors would like to thank Pietro Cicuta, Jean-François Joanny, Martin Lenz and Matthias Bussonnier for helpful discussion and Patricia Bassereau for support in the micropipet aspiration experiments. TB was supported by the ANR (ANR-11JSV5-0002). CC was supported by the ANR "Programme Blanc" (ANR-08-BLAN-0012-12 and ANR- 09-BLAN-0283). JL was supported by AXA Research Fund.

\section{References}

1 L. Pontani, J. Van der Gucht, G. Salbreux, J. Heuvingh, J. Joanny and C. Sykes, Biophys. J., 2009, 96, 192-198.

2 V. Noireaux and A. Libchaber, Proc. Natl. Acad. Sci. U. S. A., 2004, 101, 17669-17674.

3 L. Ma and S. L. Cockroft, ChemBioChem, 2010, 11, 25-34. 
4 G. Menestrina, J. Membr. Biol., 1986, 90, 177-190.

5 L. Song, Science, 1996, 274, 1859.

6 L. Movileanu, Trends Biotechnol., 2009, 27, 333341.

7 L. Mathivet, S. Cribier and P. Devaux, Biophys. J., 1996, 70, 1112-1121.

8 M. Gogler, T. Betz and J. A. Kas, Opt. Lett., 2007, 32, 18931895.

9 T. Betz, M. Lenz, J. Joanny and C. Sykes, Proc. Natl. Acad. Sci. U. S. A., 2009, 106, 15320-15325.

10 F. Gittes and C. F. Schmidt, Opt. Lett., 1998, 23, 7-9.

11 T. A. Nieminen, V. L. Y. Loke, A. B. Stilgoe, G. Knner, A. M. Braczyk, N. R. Heckenberg and H. RubinszteinDunlop, J. Opt. A: Pure Appl. Opt., 2007, 9, S196S203.
12 N. Borghi, O. Rossier and F. Brochard-Wyart, Europhys. Lett., 2003, 64, 837-843.

13 M. J. Russo, H. Bayley and M. Toner, Nat. Biotechnol., 1997, 15, 278-282.

14 J. R. Pappenheimer, E. M. Renkin and L. M. Borrero, Am. J. Physiol., 1951, 167, 13-46.

15 E. M. Renkin, J. Gen. Physiol., 1954, 38, 225-243.

16 S. G. Schultz, J. Gen. Physiol., 1961, 44, 1189-1199.

17 K. Olbrich, W. Rawicz, D. Needham and E. Evans, Biophys. J., 2000, 79, 321-327.

$18 \mathrm{~J}$. Happel and H. Brenner, Low Reynolds Number Hydrodynamics: with Special Applications to Particulate Media, Springer, 1st edn, 1983.

19 N. Borghi, S. Kremer, V. Askovic and F. Brochard-Wyart, Europhys. Lett., 2006, 75, 666-672. 